Pacific

Journal of

Mathematics

\title{
AFFINE WEAKLY REGULAR TENSOR TRIANGULATED CATEGORIES
}

IVo Dell'Ambrogio AND DonALD STANLEY 


\title{
AFFINE WEAKLY REGULAR TENSOR TRIANGULATED CATEGORIES
}

\author{
IVo DELl' Ambrogio AND DONALd STANLEY
}

\begin{abstract}
We prove that the Balmer spectrum of a tensor triangulated category is homeomorphic to the Zariski spectrum of its graded central ring, provided the triangulated category is generated by its tensor unit and the graded central ring is noetherian and regular in a weak sense. There follows a classification of all thick subcategories, and the result extends to the compactly generated setting to yield a classification of all localizing subcategories as well as the analog of the telescope conjecture. This generalizes results of Shamir for commutative ring spectra.
\end{abstract}

\section{Introduction and results}

Let $\mathcal{K}$ be an essentially small tensor triangulated category, with symmetric exact tensor product $\otimes$ and tensor unit object 1. Balmer [2005] defined a topological space, the spectrum $\mathrm{Spc} \mathcal{K}$, that allows for the development of a geometric theory of $\mathcal{K}$, similarly to how the Zariski spectrum captures the intrinsic geometry of commutative rings; see the survey [Balmer 2010b]. Among other uses, Balmer's spectrum encodes the classification of the thick tensor ideals of $\mathcal{K}$ in terms of certain subsets. It is therefore of interest to find an explicit description of the spectrum in the examples, but this is usually a difficult problem requiring some in-depth knowledge of each example at hand.

The goal of this note is to show that in some cases a concrete description of the spectrum can be obtained easily and completely formally. Let us denote by

$$
R:=\operatorname{End}_{\mathcal{K}}^{*}(\mathbf{1})=\bigoplus_{i \in \mathbb{Z}} \operatorname{Hom}_{\mathcal{K}}\left(\mathbf{1}, \Sigma^{i} \mathbf{1}\right)
$$

the graded endomorphism ring of the unit, where $\Sigma: \mathcal{K} \rightarrow \mathcal{K}$ is the suspension functor. In the terminology of [Balmer 2010a], this is the graded central ring of $\mathcal{K}$. It is a graded commutative ring and therefore we can consider its spectrum

Dell'Ambrogio was partially supported by the Labex CEMPI (ANR-11-LABX-0007-01) .

MSC2010: primary 18E30; secondary 55P42, 55U35.

Keywords: tensor triangulated category, thick subcategory, localizing subcategory, spectrum. 
of homogeneous prime ideals, Spec $R$, equipped with the Zariski topology. As established in that paper, there is always a canonical continuous map

$$
\rho: \operatorname{Spc} \mathcal{K} \rightarrow \operatorname{Spec} R
$$

comparing the two spectra. Under some mild hypotheses, e.g., when $R$ is noetherian, $\rho$ can be shown to be surjective, but it is less frequently injective and, when it is, the proof of injectivity is typically much harder.

Here is our main result:

Theorem 1.1. Assume that $\mathcal{K}$ satisfies the two following conditions:

(a) $\mathcal{K}$ is classically generated by $\mathbf{1}$, i.e., as a thick subcategory: $\operatorname{Thick}(\mathbf{1})=\mathcal{K}$.

(b) $R$ is a (graded) noetherian ring concentrated in even degrees and, for every homogeneous prime ideal $\mathfrak{p}$ of $R$, the maximal ideal of the local ring $R_{\mathfrak{p}}$ is generated by a (finite) regular sequence of homogeneous non-zero-divisors.

Then the comparison map $\rho: \operatorname{Spc} \mathcal{K} \stackrel{\sim}{\longrightarrow}$ Spec $R$ is a homeomorphism.

As in the title, we may refer to a tensor triangulated category $\mathcal{K}$ satisfying hypotheses (a) and (b) as being affine and weakly regular, respectively. Note that $R$ being noetherian implies that $R^{0}=\operatorname{End}_{\mathcal{K}}(\mathbf{1})$ is a noetherian ring and that $R$ is a finitely generated $R^{0}$-algebra, by [Goto and Yamagishi 1983].

The next result is an easy consequence of the theorem. Here $\operatorname{Supp}_{R} H^{*} X$ denotes the (big) Zariski support of the cohomology graded $R$-module $H^{*} X:=\operatorname{Hom}_{\mathcal{K}}^{*}(\mathbf{1}, X)$.

Corollary 1.2. If $\mathcal{K}$ and $R$ are as in the theorem, then there exists a canonical inclusion-preserving bijection

\section{$\{$ thick subcategories $\mathcal{C}$ of $\mathcal{K}\} \stackrel{\sim}{\rightleftarrows}$ specialization closed subsets $V$ of $\operatorname{Spec} R\}$}

mapping a thick subcategory $\mathcal{C}$ to $V=\bigcup_{X \in \mathcal{C}} \operatorname{Supp}_{R} H^{*} X$ and a specialization closed subset $V$ to $\mathcal{C}=\left\{X \in \mathcal{K} \mid \operatorname{Supp}_{R} H^{*} X \subseteq V\right\}$.

In many natural examples, $\mathcal{K}$ occurs as the subcategory $\mathcal{T}^{c}$ of compact objects in a compactly generated tensor triangulated category $\mathcal{T}$. By the latter we mean a compactly generated triangulated category $\mathcal{T}$ equipped with a symmetric monoidal structure $\otimes$ which preserves coproducts and exact triangles in both variables, and such that the compact objects form a tensor subcategory $\mathcal{T}^{c}$ (that is, $\mathbf{1}$ is compact and the tensor product of two compact objects is again compact).

In this case, the same hypotheses allow us to classify also the localizing subcategories of $\mathcal{T}$, thanks to the stratification theory of compactly generated categories due to Benson, Iyengar and Krause [Benson et al. 2011]. The support $\operatorname{supp}_{R} X \subseteq \operatorname{Spec} R$ of an object $X \in \mathcal{T}$ is defined in [Benson et al. 2008], and can be described as the set

$$
\operatorname{supp}_{R} X=\{\mathfrak{p} \in \operatorname{Spec} R \mid X \otimes K(\mathfrak{p}) \neq 0\},
$$


where the residue field object $K(\mathfrak{p})$ of a prime ideal $\mathfrak{p}$ is an object whose cohomology is the graded residue field of $R$ at $\mathfrak{p}$; see Section 3.

Theorem 1.3. Let $\mathcal{T}$ be a compactly generated tensor triangulated category with compact objects $\mathcal{K}:=\mathcal{T}^{c}$ and graded central ring $R$ satisfying conditions $(a)$ and $(b)$. Then we have the following canonical inclusion-preserving bijection:

$$
\text { \{localizing subcategories } \mathcal{L} \subseteq \mathcal{T}\} \stackrel{\sim}{\longleftrightarrow} \text { subsets } S \subseteq \operatorname{Spec} R\} .
$$

The correspondence sends a localizing subcategory $\mathcal{L}$ to $S=\bigcup_{X \in \mathcal{L}} \operatorname{supp}_{R} X$, and an arbitrary subset $S$ to $\mathcal{L}=\left\{X \in \mathcal{T} \mid \operatorname{supp}_{R} X \subseteq S\right\}$. Moreover, the bijection restricts to localizing subcategories $\mathcal{L}=\operatorname{Loc}(\mathcal{L} \cap \mathcal{K})$ which are generated by compact objects on the left and to specialization closed subsets $S=\bigcup_{\mathfrak{p} \in S} V(\mathfrak{p})$ on the right.

Note that here the affine condition (a) is equivalent to requiring that $\mathcal{T}$ is generated by 1 as a localizing subcategory. As $\operatorname{Supp}_{R} H^{*} X=\operatorname{supp}_{R} X$ for all compact objects $X \in \mathcal{K}$, one sees easily that in the compactly generated case Theorem 1.1 and Corollary 1.2 are also a consequence of Theorem 1.3.

The next corollary is another byproduct of stratification. Recall that a localizing subcategory $\mathcal{L} \subseteq \mathcal{T}$ is smashing if the inclusion functor $\mathcal{L} \hookrightarrow \mathcal{T}$ admits a coproductpreserving right adjoint.

Corollary 1.4 (the telescope conjecture in the affine weakly regular case). In the situation of Theorem 1.3, every smashing subcategory of $\mathcal{T}$ is generated by a set of compact objects of $\mathcal{T}$.

A few special cases of our formal results had already been observed, such as when $R$ is even periodic and of global dimension at most one; see [Dell'Ambrogio and Tabuada 2012]. We now consider some more concrete examples.

$$
* * *
$$

Example 1.5. Let $A$ be a commutative dg algebra and $D(A)$ its derived category of dg modules. Then $D(A)$ is an affine compactly generated tensor triangulated category with respect to the standard tensor product $\otimes=\otimes_{A}^{\mathrm{L}}$, and $R=H^{*} A$ is the cohomology algebra of $A$; thus if the latter satisfies (b) all our results apply to $D(A)$. Actually, in this example we can improve our results a little by eliminating the hypothesis that $R$ is even and that the elements of the regular sequences are non-zero-divisors:

Theorem 1.6. Let A be a commutative dg algebra such that its graded cohomology ring $R=H^{*} A$ is noetherian and such that every local prime $\mathfrak{p} R_{\mathfrak{p}}$ is generated by a finite regular sequence. Then all the conclusions of Theorems 1.1 and 1.3 and of Corollaries 1.2 and 1.4 hold for $\mathcal{T}=D(A)$ and $\mathcal{K}=D(A)^{c}$.

We can apply this, for instance, to a graded polynomial algebra with any choice of grading for the variables, seen as a strictly commutative formal dg algebra. 
Example 1.7. Let $A$ be a commutative $S$-algebra (a.k.a. commutative highly structured ring spectrum), and let $D(A)$ be its derived category. (This covers Example 1.5, as commutative dga's can be seen as commutative $S$-algebras.) Then $D(A)$ is an affine compactly generated tensor triangulated category, and $R=\pi_{*} A$ is the stable homotopy algebra of $A$; thus if the latter satisfies (b) all our results apply to $D(A)$. Shamir [2012] already treated this example under the additional hypothesis that $\pi_{*} A$ has finite Krull dimension. Working with $\infty$-categories and $\mathrm{E}_{\infty}$-rings, Mathew [2015, Theorem 1.4] established the classification of thick subcategories as in Corollary 1.2 for the case when $\pi_{*} A$ is even periodic and $\pi_{0} A$ regular noetherian. Remarkably, in the special case of $S$-algebras defined over $\mathbb{Q}$, Mathew was also able to prove the classification of thick subcategories only assuming $\pi_{*} A$ noetherian, i.e., without any regularity hypothesis; see [Mathew 2016, Theorem 1.4]. (Note however that, thanks to [Mandell 2012], in order to apply our own results we really only need an $\mathrm{E}_{4}$-structure on a ring spectrum rather than a fully commutative $\mathrm{E}_{\infty}$-structure.)

The next two well-known examples show that neither hypothesis (a) nor (b) can be weakened with impunity.

Example 1.8. The derived category $\mathcal{T}=D\left(\mathbb{P}_{k}^{1}\right)$ of the projective line over a field $k$ is an example where $R=\operatorname{End}^{*}(\mathbf{1}) \simeq k$ certainly satisfies (b) but (a) does not hold. Indeed $\rho$ can be identified with the structure map $\mathbb{P}_{k}^{1} \rightarrow \operatorname{Spec} k$ and is therefore far from injective in this case; see [Balmer 2010a, Remark 8.2].

Example 1.9. If $\mathcal{T}=D(A)$ is the derived category of a commutative (ungraded) ring $A$, Theorem 1.1 and the classification of thick subcategories always hold by a result of Thomason [1997] (see [Balmer 2010a, Proposition 8.1]); the classification of localizing subcategories and the telescope conjecture hold if $A$ is noetherian by [Neeman 1992a]. On the other hand, Keller [1994] found examples of nonnoetherian rings $A$ for which the two latter results fail.

In view of these examples, it would be interesting to know how far our weak regularity hypothesis (b) can be weakened in general. Would noetherian suffice?

\section{Preliminaries}

Let $\mathcal{K}$ be an essentially small tensor triangulated category.

For any two objects $X, Y \in \mathcal{K}$, consider the $\mathbb{Z}$-graded group $\operatorname{Hom}_{\mathcal{K}}^{*}(X, Y)=$ $\bigoplus_{i \in \mathbb{Z}} \operatorname{Hom}_{\mathcal{K}}\left(X, \Sigma^{i} Y\right)$. Recall that the symmetric tensor product of $\mathcal{K}$ canonically induces on $R:=\operatorname{Hom}_{\mathcal{K}}^{*}(\mathbf{1}, \mathbf{1})$ the structure of a graded commutative ${ }^{1}$ ring, and

\footnotetext{
${ }^{1}$ To be precise, graded commutativity means here that $f g=\epsilon^{|f|}|g| g f$ for any two homogeneous elements $f \in \operatorname{Hom}_{\mathcal{K}}\left(\mathbf{1}, \Sigma^{|f|} \mathbf{1}\right)$ and $g \in \operatorname{Hom}_{\mathcal{K}}\left(\mathbf{1}, \Sigma^{|g|} \mathbf{1}\right)$, where $\epsilon \in R^{0}$ is a constant with $\epsilon^{2}=1$ induced by the symmetry isomorphism $\Sigma 1 \otimes \Sigma 1 \stackrel{\sim}{\longrightarrow} \Sigma 1 \otimes \Sigma 1$. In most cases we have $\epsilon=-1$, e.g., if $\mathcal{K}$ admits a symmetric monoidal model, but usually no extra difficulty arises by allowing the general case. Of course, this is immaterial for $R$ even.
} 
on each $\operatorname{Hom}_{\mathcal{K}}^{*}(X, Y)$ the structure of a (left and right) graded $R$-module. The composition of maps in $\mathcal{K}$ and the tensor functor $-\otimes-$ are (graded) bilinear for this action. See [Balmer 2010a, §3] for details.

Since we are using cohomological gradings, we write $H^{*} X$ for the $R$-module $\operatorname{Hom}_{\mathcal{K}}^{*}(\mathbf{1}, X)$ and call it the cohomology of $X$.

Supports for graded modules. We denote by Spec $R$ the Zariski spectrum of all homogeneous prime ideals in $R$. If $M$ is an $R$-module (always understood to be graded) and $\mathfrak{p} \in \operatorname{Spec} R$, the graded localization of $M$ at $\mathfrak{p}$ is the $R$-module $M_{\mathfrak{p}}$ obtained by inverting the action of all the homogeneous elements in $R \backslash \mathfrak{p}$. The big support of $M$ is the following subset of the spectrum:

$$
\operatorname{Supp}_{R} M=\left\{\mathfrak{p} \in \operatorname{Spec} R \mid M_{\mathfrak{p}} \neq 0\right\} .
$$

Since our graded ring $R$ is noetherian we also dispose of the small support, defined in terms of the indecomposable injective $R$-modules $E(R / \mathfrak{p})$ :

$\operatorname{supp}_{R} M=\{\mathfrak{p} \mid E(R / \mathfrak{p})$ occurs in the minimal injective resolution of $M\}$.

We recall from [Benson et al. 2008, §2] some well-known properties of supports. In general we have $\operatorname{supp}_{R} M \subseteq \operatorname{Supp}_{R} M$. If $M$ is finitely generated, these two sets are equal and also coincide with the Zariski closed set $V\left(\operatorname{Ann}_{R} M\right)$. For a general $M, \operatorname{Supp}_{R} M$ is always specialization closed: if it contains any point $\mathfrak{p}$ then it must contain its closure $V(\mathfrak{p})=\{\mathfrak{q} \mid \mathfrak{p} \subseteq \mathfrak{q}\}$. In fact $\operatorname{Supp}_{R} M$ is equal to the specialization closure of $\operatorname{supp}_{R} M: \operatorname{Supp}_{R} M=\bigcup_{\mathfrak{p} \in \operatorname{supp}_{R} M} V(\mathfrak{p})$. The small support plays a fundamental role in the Benson-Iyengar-Krause stratification theory, but in this note it will only appear implicitly.

The next lemma follows by a standard induction on the length of the objects.

Lemma 2.1. If $\mathcal{K}=$ Thick(1) is affine and $R$ is noetherian, the graded $R$-module $\operatorname{Hom}_{\mathcal{K}}^{*}(X, Y)$ is finitely generated for all $X, Y \in \mathcal{K}$.

The comparison map of spectra. Recall from [Balmer 2005] that, as a set, the spectrum $\operatorname{Spc} \mathcal{K}$ is defined to be the collection of all proper thick subcategories $\mathcal{P} \subsetneq \mathcal{K}$ which are prime tensor ideals: $X \otimes Y \in \mathcal{P} \Longleftrightarrow X \in \mathcal{P}$ or $Y \in \mathcal{P}$. For every $\mathcal{P} \in \operatorname{Spc} \mathcal{K}$, let $\rho_{\mathcal{K}}(\mathcal{P})$ denote the ideal of Spec $R$ generated by the set of homogeneous elements $\left\{f: \mathbf{1} \rightarrow \Sigma^{|f|} \mathbf{1} \mid \operatorname{cone}(f) \notin \mathcal{P}\right\}$. By [Balmer 2010a, Theorem 5.3], the assignment $\mathcal{P} \mapsto \rho_{\mathcal{K}}(\mathcal{P})$ defines a continuous map $\rho_{\mathcal{K}}: \mathrm{Spc} \mathcal{K} \rightarrow \operatorname{Spec} R$, natural in $\mathcal{K}$. Moreover, the two spaces $\operatorname{Spc} \mathcal{K}$ and Spec $R$ are spectral in the sense of Hochster [1969], and $\rho_{\mathcal{K}}$ is a spectral map in that the preimage of a compact open set is again compact.

Lemma 2.2. If $\rho_{\mathcal{K}}$ is bijective then it is a homeomorphism. 
Proof. This is an immediate consequence of [Hochster 1969, Proposition 15], which says that for a spectral map of spectral topological spaces to be a homeomorphism it suffices that it is an order isomorphism for the specialization order of the two spaces. Recall that the specialization order is defined for the points of any topological space by $x \geq y \Longleftrightarrow x \in \overline{\{y\}}$. Indeed $\rho:=\rho_{\mathcal{K}}$ is inclusion reversing, $\mathcal{Q} \subseteq \mathcal{P} \Longleftrightarrow \rho(\mathcal{Q}) \supseteq \rho(\mathcal{P})$, hence it maps the closure $\overline{\{\mathcal{P}\}}=\{\mathcal{Q} \mid \mathcal{Q} \subseteq \mathcal{P}\}$ in Spc $\mathcal{K}$ of any point $\mathcal{P}$ to the Zariski closure $V(\rho(\mathcal{P}))=\{\mathfrak{q} \mid \mathfrak{q} \supseteq \rho(\mathcal{P})\}$ in Spec $R$ of the corresponding point.

Central localization. For every prime ideal $\mathfrak{p}$ of the graded central ring $R$ of $\mathcal{K}$, there exists by [Balmer 2010a, Theorem 3.6] a tensor triangulated category $\mathcal{K}_{\mathfrak{p}}$ having the same objects as $\mathcal{K}$ and such that its graded Hom modules are the localizations

$$
\operatorname{Hom}_{\mathcal{K}_{\mathfrak{p}}}^{*}(X, Y)=\operatorname{Hom}_{\mathcal{K}}^{*}(X, Y)_{\mathfrak{p}}
$$

In particular the graded central ring of $\mathcal{K}_{\mathfrak{p}}$ is the local ring $R_{\mathfrak{p}}$. There is a canonical exact functor $q_{\mathfrak{p}}: \mathcal{K} \rightarrow \mathcal{K}_{\mathfrak{p}}$, which is in fact the Verdier quotient by the thick tensor ideal generated by $\{$ cone $(f) \in \mathcal{K} \mid f \in R \backslash \mathfrak{p}$ homogeneous $\}$. For emphasis, we will sometimes write $X_{\mathfrak{p}}$ for $X=q_{\mathfrak{p}} X$ when considered as an object of $\mathcal{K}_{\mathfrak{p}}$.

Clearly if $\mathcal{K}$ is generated by $\mathbf{1}$ then $\mathcal{K}_{\mathfrak{p}}$ is generated by $\mathbf{1}_{\mathfrak{p}}$. Later we will use the fact that if a tensor triangulated category is generated by its unit then every thick subcategory is automatically a tensor ideal.

Let $\ell_{\mathfrak{p}}: R \rightarrow R_{\mathfrak{p}}$ denote the localization map between the graded central rings of the two categories. By [Balmer 2010a, Theorem 5.4], we have a pullback square of spaces

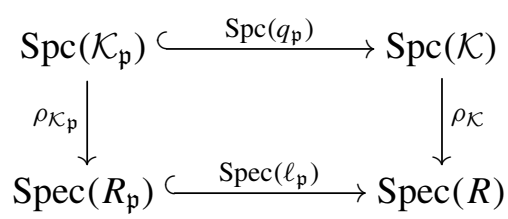

where the horizontal maps are injective.

Koszul objects. We adapt some convenient notation from [Benson et al. 2008]. For any object $X \in \mathcal{K}$ and homogeneous element $f \in R$, let $X / / f:=\operatorname{cone}(f \cdot X)$ be any choice of mapping cone for the map $f \cdot X: \Sigma^{-|f|} X \rightarrow X$ given by the $R$-action. If $f_{1}, \ldots, f_{n}$ is a finite sequence of homogeneous elements, define recursively $X_{0}:=X$ and $X_{i}:=X / /\left(f_{1}, \ldots, f_{i}\right):=\left(X / /\left(f_{1}, \ldots, f_{i-1}\right)\right) / / f_{i}$ for $i \in\{1, \ldots, n\}$. Thus by construction we have exact triangles

$$
\Sigma^{-\left|f_{i}\right|} X_{i-1} \stackrel{f_{i} \cdot X_{i-1}}{\longrightarrow} X_{i-1} \longrightarrow X_{i} \longrightarrow \Sigma^{-\left|f_{i}\right|+1} X_{i-1},
$$


and moreover, since the tensor product is exact, we have isomorphisms

$$
X / /\left(f_{1}, \ldots, f_{i}\right) \simeq X \otimes \mathbf{1} / / f_{1} \otimes \cdots \otimes \mathbf{1} / / f_{i}
$$

for all $i \in\{1, \ldots, n\}$. In the following, we will perform this construction inside the $\mathfrak{p}$-local category $\mathcal{K}_{\mathfrak{p}}$.

We need the following triangular version of the Nakayama lemma, for $\mathcal{K}$ affine.

Lemma 2.5. If $X \in \mathcal{K}_{\mathfrak{p}}$ is any object and $f_{1}, \ldots, f_{n}$ is a set of homogeneous generators for $\mathfrak{p} R_{\mathfrak{p}}$, then in $\mathcal{K}_{\mathfrak{p}}$ we have $X=0$ if and only if $X / /\left(f_{1}, \ldots, f_{n}\right)=0$.

Proof. Since $\mathcal{K}$ and thus $\mathcal{K}_{\mathfrak{p}}$ are generated by their tensor unit, it suffices to show that $H^{*} X_{\mathfrak{p}}=0$ if and only if $H^{*}\left(X / /\left(f_{1}, \ldots, f_{n}\right)\right)_{\mathfrak{p}}=0$, and the latter can be proved as in [Benson et al. 2008, Lemma 5.11 (3)]. We give the easy argument for completeness.

With the above notation, by taking cohomology $H^{*}=\operatorname{Hom}_{\mathcal{K}_{\mathfrak{p}}}^{*}\left(\mathbf{1}_{\mathfrak{p}},-\right)$ of the triangle (2.4) of $\mathcal{K}_{\mathfrak{p}}$ we obtain the long exact sequence of $R_{\mathfrak{p}}$-modules

$$
\cdots \longrightarrow H^{*-\left|f_{i}\right|} X_{i-1} \stackrel{f_{i}}{\longrightarrow} H^{*} X_{i-1} \longrightarrow H^{*} X_{i} \longrightarrow H^{*-\left|f_{i}\right|+1} X_{i-1} \longrightarrow \cdots,
$$

where each module is finitely generated by Lemma 2.1. Since $f_{i} \in \mathfrak{p}$, if $H^{*} X_{i-1} \neq 0$ the first map in the sequence is not invertible by the Nakayama lemma, hence $H^{*} X_{i} \neq 0$. The evident recursion shows that $H^{*} X \neq 0$ implies $H^{*} X_{n} \neq 0$. The very same exact sequences also show that if $H^{*} X=0$ then $H^{*} X_{n}=0$.

\section{Thick subcategories}

Assume from now on that $\mathcal{K}$ satisfies conditions (a) and (b) of Theorem 1.1.

Residue field objects. By hypothesis, for every prime ideal $\mathfrak{p} \in \operatorname{Spec} R$ there exists a regular sequence $f_{1}, \ldots, f_{n}$ of homogeneous non-zero-divisors of $R_{\mathfrak{p}}$ which generate the ideal $\mathfrak{p} R_{\mathfrak{p}}$. Choose one such sequence once and for all, and construct the associated Koszul object

$$
K(\mathfrak{p}):=\mathbf{1}_{\mathfrak{p}} / /\left(f_{1}, \ldots, f_{n}\right) \simeq \mathbf{1}_{\mathfrak{p}} / / f_{1} \otimes \cdots \otimes \mathbf{1}_{\mathfrak{p}} / / f_{n}
$$

in the $\mathfrak{p}$-local tensor triangulated category $\mathcal{K}_{\mathfrak{p}}$.

Lemma 3.1. For every object $X \in \mathcal{K}_{\mathfrak{p}}$ and every $i \in\{1, \ldots, n\}$, each element $f$ of the ideal $\left(f_{1}, \ldots, f_{i}\right) \subset R_{\mathfrak{p}}$ acts as zero on $X / /\left(f_{1}, \ldots, f_{i}\right)$, i.e., $f \cdot X / /\left(f_{1}, \ldots, f_{i}\right)=0$.

Proof. Recall that, as an immediate consequence of the $R_{\mathfrak{p}}$-bilinearity of the composition in $\mathcal{K}_{\mathfrak{p}}$, the elements of $R_{\mathfrak{p}}$ acting as zero on an object $Y$ form an ideal (coinciding with the annihilator of the $R_{\mathfrak{p}}$-module $\operatorname{Hom}_{\mathcal{K}_{\mathfrak{p}}}^{*}(Y, Y)$ ). Thanks to the isomorphism $X / /\left(f_{1}, \ldots, f_{i}\right) \simeq X \otimes \mathbf{1}_{\mathfrak{p}} / / f_{1} \otimes \cdots \otimes \mathbf{1}_{\mathfrak{p}} / / f_{i}$ and the $R_{\mathfrak{p}}$-linearity of 
the tensor product, it will therefore suffice to prove that $f_{i}$ acts as zero on $\mathbf{1}_{\mathfrak{p}} / / f_{i}$. Consider the commutative diagram

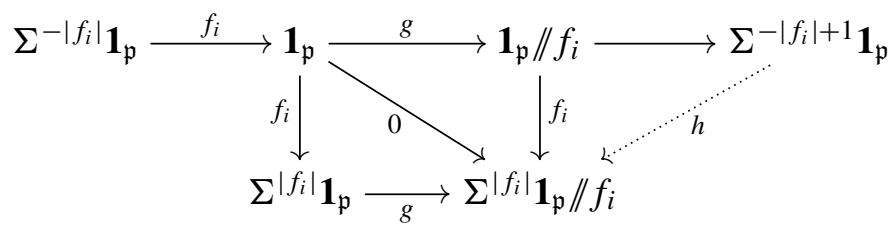

where the top row is the exact triangle defining $\mathbf{1}_{\mathfrak{p}} / / f_{i}$. Being the composite of two consecutive maps in a triangle, $g f_{i}$ is zero. Up to a suspension, this is also the diagonal map in the square. Hence $f_{i} \cdot \mathbf{1}_{\mathfrak{p}} / / f_{i}$ factors through a map $h$ as pictured. Since $R$ is even by hypothesis, we have that $R_{\mathfrak{p}}$ is even, and we claim that also

$$
H^{n}\left(\mathbf{1}_{\mathfrak{p}} / / f_{i}\right)=0 \quad \text { for all odd } n .
$$

This implies $h=0$ and therefore $f_{i} \cdot \mathbf{1}_{\mathfrak{p}} / / f_{i}=0$, as required. To prove the claim, note that the defining triangle of $\mathbf{1}_{\mathfrak{p}} / / f_{i}$ induces the exact sequence

$$
R_{\mathfrak{p}}^{n-\left|f_{i}\right|} \stackrel{f_{i}}{\longrightarrow} R_{\mathfrak{p}}^{n} \longrightarrow H^{n}\left(\mathbf{1}_{\mathfrak{p}} / / f_{i}\right) \longrightarrow R_{\mathfrak{p}}^{n-\left|f_{i}\right|+1} \stackrel{f_{i}}{\longrightarrow} R_{\mathfrak{p}}^{n+1},
$$

where the first and last maps are injective by the hypothesis that $f_{i}$ is a non-zerodivisor in $R_{\mathfrak{p}}$. Thus (3.2), and even $H^{*}\left(\mathbf{1}_{\mathfrak{p}} / / f_{i}\right) \simeq R_{\mathfrak{p}} /\left(f_{i}\right)$, follows immediately.

Corollary 3.3. $H^{*}(X \otimes K(\mathfrak{p}))$ is a graded $k(\mathfrak{p})$-vector space for every $X \in \mathcal{K}_{\mathfrak{p}}$.

Proof. By Lemma 3.1 together with the $R$-linearity of the tensor product, each $f \in \mathfrak{p} R_{\mathfrak{p}}$ acts as zero on $X \otimes K(\mathfrak{p}) \simeq X \otimes \mathbf{1}_{\mathfrak{p}} / /\left(f_{1}, \ldots, f_{n}\right)$. Therefore all such $f$ also act as zero on $H^{*}(X \otimes K(\mathfrak{p}))$ by the $R$-linearity of composition.

Lemma 3.4. There is an isomorphism $H^{*}\left(\mathbf{1}_{\mathfrak{p}} / /\left(f_{1}, \ldots, f_{i}\right)\right) \simeq R_{\mathfrak{p}} /\left(f_{1}, \ldots, f_{i}\right)$ of $R$-modules for all $i \in\{1, \ldots, n\}$. In particular $H^{*} K(\mathfrak{p})$ is isomorphic to the residue field $k(\mathfrak{p}):=R_{\mathfrak{p}} / \mathfrak{p} R_{\mathfrak{p}}$.

Proof. Write $C_{0}=\mathbf{1}_{\mathfrak{p}}$ and $C_{i}:=\mathbf{1}_{\mathfrak{p}} / /\left(f_{1}, \ldots, f_{i}\right)$ for short. Then $K(\mathfrak{p})=C_{n}$, and for all $i \in\{1, \ldots, n\}$ we have exact triangles

$$
\Sigma^{-\left|f_{i}\right|} C_{i-1} \stackrel{f_{i} \cdot C_{i-1}}{\longrightarrow} C_{i-1} \longrightarrow C_{i} \longrightarrow \Sigma^{-\left|f_{i}\right|+1} C_{i-1} .
$$

The claim follows by recursion on $i$. Indeed $H^{*} C_{0}=R_{\mathfrak{p}}$, and assume that $H^{*} C_{i-1} \simeq$ $R_{\mathfrak{p}} /\left(f_{1}, \ldots, f_{i-1}\right)$. Then the above triangle induces an exact sequence

$$
H^{*-\left|f_{i}\right|} C_{i-1} \stackrel{f_{i}}{\longrightarrow} H^{*} C_{i-1} \longrightarrow H^{*} C_{i} \longrightarrow H^{*-\left|f_{i}\right|+1} C_{i-1} \stackrel{f_{i}}{\longrightarrow} H^{*+1} C_{i-1},
$$

where the first and last maps are injective because by hypothesis $f_{i}$ is a nonzero-divisor in the ring $R_{\mathfrak{p}} /\left(f_{1}, \ldots, f_{i-1}\right)$. We thus obtain a short exact sequence $0 \rightarrow f_{i} R_{\mathfrak{p}} /\left(f_{1}, \ldots, f_{i-1}\right) \rightarrow R_{\mathfrak{p}} /\left(f_{1}, \ldots, f_{i-1}\right) \rightarrow H^{*} C_{i} \rightarrow 0$, proving the claim for $i$. 
Remark 3.5. Of the weak regularity hypothesis (b), the proof of Lemma 3.4 only uses that $f_{1}, \ldots, f_{n}$ is a regular sequence, while the proof of Lemma 3.1 only uses that the $f_{i}$ are non-zero-divisors in $R_{\mathfrak{p}}$ and that the ring $R$ is even. These are the only places where we make use of these assumptions (the noetherian hypothesis, on the other hand, will be needed on several occasions). Note that, although we already know by Corollary 3.3 that $H^{*} K(\mathfrak{p})$ is a $k(\mathfrak{p})$-vector space, for the next proposition we also need it to be one-dimensional as per Lemma 3.4.

Proposition 3.6. For all $\mathfrak{p} \in \operatorname{Spec} R$ and $X \in \mathcal{K}_{\mathfrak{p}}$, the tensor product $X \otimes K(\mathfrak{p})$ decomposes into a coproduct of shifted copies of the residue field object:

$$
\coprod_{\alpha} \Sigma^{n_{\alpha}} K(\mathfrak{p}) \stackrel{\sim}{\longrightarrow} X \otimes K(\mathfrak{p}) .
$$

Proof. By Corollary 3.3 we know that $H^{*}(X \otimes K(\mathfrak{p}))$ is a graded $k(\mathfrak{p})$-vector space. Choose a graded basis $\left\{x_{\alpha}\right\}_{\alpha}$, corresponding to a morphism $\bigsqcup_{\alpha} \Sigma^{n_{\alpha}} \mathbf{1}_{\mathfrak{p}} \rightarrow X \otimes K(\mathfrak{p})$. We will show that this map extends nontrivially to the Koszul object

$$
\left(\coprod_{\alpha} \Sigma^{n_{\alpha}} \mathbf{1}_{\mathfrak{p}}\right) / /\left(f_{1}, \ldots, f_{n}\right)=\coprod_{\alpha}\left(\Sigma^{n_{\alpha}} \mathbf{1}_{\mathfrak{p}} / /\left(f_{1}, \ldots, f_{n}\right)\right) .
$$

For this, it will suffice to extend each individual map $x_{\alpha}: \Sigma^{n_{\alpha}} \mathbf{1}_{\mathfrak{p}} \rightarrow X \otimes K(\mathfrak{p})$. As before, we proceed recursively along the regular sequence $f_{1}, \ldots, f_{n}$. Consider the commutative diagram

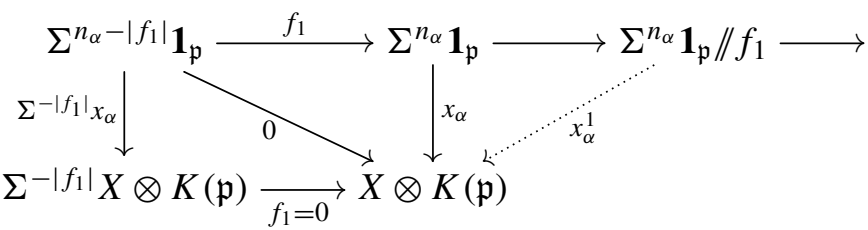

where the top row is a rotation of the defining triangle for $\mathbf{1}_{\mathfrak{p}} / / f_{1}$. The left-bottom composite vanishes because $f_{1}$ acts trivially on $X \otimes K(\mathfrak{p})$ by Lemma 3.1. Hence we obtain the map $x_{\alpha}^{1}$ on the right. Note that $x_{\alpha}^{1} \neq 0$ because $x_{\alpha} \neq 0$. Now we repeat the procedure for $i=2, \ldots, n$, using the triangle

$$
\Sigma^{-\left|f_{i}\right|} \mathbf{1}_{\mathfrak{p}} / /\left(f_{1}, \ldots, f_{i-1}\right) \stackrel{f_{i}}{\longrightarrow} \mathbf{1}_{\mathfrak{p}} / /\left(f_{1}, \ldots, f_{i-1}\right) \longrightarrow \mathbf{1}_{\mathfrak{p}} / /\left(f_{1}, \ldots, f_{i}\right) \longrightarrow
$$

to extend $x_{\alpha}^{i-1}$ to a nonzero map $x_{\alpha}^{i}: \Sigma^{n_{\alpha}} \mathbf{1}_{\mathfrak{p}} / /\left(f_{1}, \ldots, f_{i}\right) \rightarrow X \otimes K(\mathfrak{p})$ hitting the same element in cohomology. In particular we obtain the announced extension $x_{\alpha}^{n}: \Sigma^{n_{\alpha}} K(\mathfrak{p}) \rightarrow X \otimes K(\mathfrak{p})$. As a nonzero map on a one-dimensional $k(\mathfrak{p})$-vector space (Lemma 3.4), the induced map $H^{*}\left(x_{\alpha}^{n}\right)$ must be injective. Hence, collectively, the maps $\left\{x_{\alpha}^{n}\right\}_{\alpha}$ yield an isomorphism as required.

Proposition 3.7. For every $\mathfrak{p}$, the thick subcategory $\operatorname{Thick}(K(\mathfrak{p}))$ of $\mathcal{K}_{\mathfrak{p}}$ is minimal, meaning that it contains no proper nonzero thick subcategories. 
Proof. Note that for every nonzero object $X$ of $\mathcal{K}_{\mathfrak{p}}$ we have $X \otimes K(\mathfrak{p}) \neq 0$. Indeed if $X \otimes K(\mathfrak{p})=X / /\left(f_{1}, \ldots, f_{n}\right)=0$ then $X_{\mathfrak{p}}=0$ by Lemma 2.5.

Let $\mathcal{C}$ be a thick subcategory of $\operatorname{Thick}(K(\mathfrak{p}))$. Because $\mathcal{C}$ is a tensor ideal, if it contains a nonzero object $X$ then it also contains $X \otimes K(\mathfrak{p})$, which is again nonzero by the above observation. Therefore $\mathcal{C}$ must contain a shifted copy of $K(\mathfrak{p})$ by Proposition 3.6, hence $\mathcal{C}=\operatorname{Thick}(K(\mathfrak{p}))$.

Proof of Theorem 1.1. Now we show how to deduce our main result from the minimality of the thick subcategories Thick $(K(\mathfrak{p}))$. By Lemma 2.2 it will suffice to show that the map $\rho_{\mathcal{K}}: \operatorname{Spc} \mathcal{K} \rightarrow \operatorname{Spec} R$ is bijective. Since $R$ is graded noetherian, $\rho_{\mathcal{K}}$ is surjective by [Balmer 2010a, Theorem 7.3]. It remains to prove it is injective.

Let $\mathfrak{p} \in \operatorname{Spec} R$ be any homogeneous prime. We must show that the fiber of the comparison map $\rho_{\mathcal{K}}: \operatorname{Spc} \mathcal{K} \rightarrow \operatorname{Spec} R$ over $\mathfrak{p}$ consists of a single prime tensor ideal. By the pullback square (2.3), every point of Spc $\mathcal{K}$ lying over $\mathfrak{p}$ must belong to $\operatorname{Spc} \mathcal{K}_{\mathfrak{p}}$. Hence it will suffice to show that the fiber of $\rho:=\rho_{\mathcal{K}_{\mathfrak{p}}}$ over the maximal ideal $\mathfrak{m}:=\mathfrak{p} R_{\mathfrak{p}}$ of $R_{\mathfrak{p}}$ consists of a single point. In fact a stronger statement is true: if $\mathcal{P} \in \operatorname{Spc} \mathcal{K}_{\mathfrak{p}}$ is such that $\rho(\mathcal{P})=\mathfrak{m}$, then $\mathcal{P}=\{0\}$. Let us prove this.

By definition of the comparison map we have

$$
\rho(\mathcal{P})=\left\langle\left\{f \in R_{\mathfrak{p}} \mid f \text { is homogeneous and } \mathbf{1}_{\mathfrak{p}} / / f \notin \mathcal{P}\right\}\right\rangle,
$$

and as $\rho(\mathcal{P}) \subseteq \mathfrak{m}$ always holds by the maximality of $\mathfrak{m}$, the hypothesis $\rho(\mathcal{P})=\mathfrak{m}$ precisely means that $\mathbf{1}_{\mathfrak{p}} / / f \notin \mathcal{P}$ for all homogeneous elements $f \in \mathfrak{m}$. In particular $\mathbf{1}_{\mathfrak{p}} / / f_{i} \notin \mathcal{P}$ for the elements $f_{i}$ in the chosen regular sequence for $\mathfrak{m}$. As $\mathcal{P}$ is a tensor prime, we deduce further that

$$
K(\mathfrak{p}) \simeq \mathbf{1}_{\mathfrak{p}} / / f_{1} \otimes \cdots \otimes \mathbf{1}_{\mathfrak{p}} / / f_{n} \notin \mathcal{P} .
$$

Now let $X \in \mathcal{P}$ and assume that $X \neq 0$. Then $X \otimes K(\mathfrak{p}) \neq 0$ by Lemma 2.5, hence

$$
\operatorname{Thick}(X \otimes K(\mathfrak{p}))=\operatorname{Thick}(K(\mathfrak{p}))
$$

by the minimality of $\operatorname{Thick}(K(\mathfrak{p}))$, Proposition 3.7. As $\mathcal{P}$ is a thick tensor ideal we also have $X \otimes K(\mathfrak{p}) \in \mathcal{P}$ and therefore $K(\mathfrak{p}) \in \mathcal{P}$ by (3.9), but this contradicts (3.8). Therefore $X=0$ and we conclude that $\mathcal{P}=\{0\}$, proving the claim. This concludes the proof of Theorem 1.1.

Proof of Corollary 1.2. To deduce Corollary 1.2 from the theorem, we must verify that the homeomorphism $\rho_{\mathcal{K}}$ identifies $\operatorname{Supp}_{R} H^{*} X \subseteq \operatorname{Spec} R$, the ring-theoretic support of an object $X \in \mathcal{K}$, with $\operatorname{supp} X:=\{\mathcal{P} \in \operatorname{Spc} \mathcal{K} \mid X \notin \mathcal{P}\}$, the universal support datum of $X$ :

Lemma 3.10. We have $\operatorname{Supp}_{R} H^{*} X=\rho_{\mathcal{K}}(\operatorname{supp} X)$ for all $X \in \mathcal{K}$. 
Proof. Let $\mathfrak{p}=\rho_{\mathcal{K}}(\mathcal{P})$. It follows from (2.3) that $X \in \mathcal{P}$ if and only if $X_{\mathfrak{p}} \in \mathcal{P}_{\mathfrak{p}}$, where $\mathcal{P}_{\mathfrak{p}}$ denotes $\mathcal{P}$ seen as an element of $\operatorname{Spc} \mathcal{K}_{\mathfrak{p}}$. We have just proved that $\rho_{\mathcal{K}_{\mathfrak{p}}}:$ Spc $\mathcal{K}_{\mathfrak{p}} \stackrel{\sim}{\longrightarrow} R_{\mathfrak{p}}$ is a bijection sending $\{0\}$ to $\mathfrak{p} R_{\mathfrak{p}}$, so we must have $\mathcal{P}_{\mathfrak{p}}=\{0\}$. Therefore

$$
\mathfrak{p} \in \operatorname{Supp}_{R} H^{*} X \Longleftrightarrow H^{*} X_{\mathfrak{p}} \neq 0 \Longleftrightarrow X_{\mathfrak{p}} \neq 0 \Longleftrightarrow X_{\mathfrak{p}} \notin \mathcal{P}_{\mathfrak{p}} \Longleftrightarrow \mathcal{P} \in \operatorname{supp} X
$$

Now it suffices to appeal to the abstract classification theorem [Balmer 2005, Theorem 4.10]. Indeed, since $R$ is noetherian, the space $\operatorname{Spec} R$ is noetherian and therefore its specialization closed subsets and its Thomason subsets coincide (cf. [Balmer 2005, Remark 4.11]). Moreover, since $\mathcal{K}$ is generated by its tensor unit, all its objects are dualizable (because dualizable objects form a thick subcategory and $\mathbf{1}$ is dualizable) and therefore all its thick tensor ideals are radical (see [Balmer 2007, Proposition 2.4]). Hence by Theorem 1.1 and Lemma 3.10 the classification of [Balmer 2005, Theorem 4.10] immediately translates into the classification described in Corollary 1.2, as wished.

\section{Localizing subcategories}

Assume from now on that $\mathcal{T}$ is a compactly generated tensor triangulated category such that its subcategory $\mathcal{K}:=\mathcal{T}^{c}$ of compact objects satisfies hypotheses (a) and (b) of Theorem 1.1. Thus in particular $\mathcal{T}$ is generated as a localizing subcategory by the tensor unit: $\operatorname{Loc}(\mathbf{1})=\mathcal{T}$. It follows that every localizing subcategory of $\mathcal{T}$ is automatically a tensor ideal.

Since $\mathcal{T}$ is compactly generated, the (Verdier) $\mathfrak{p}$-localization functor $q_{\mathfrak{p}}: \mathcal{K} \rightarrow \mathcal{K}_{\mathfrak{p}}$ we used so far can be extended to a finite (Bousfield) localization functor

$$
(-)_{\mathfrak{p}}: \mathcal{T} \rightarrow \mathcal{T}
$$

We briefly recall its properties, referring for all proofs to [Benson et al. 2011, §2] or [Dell'Ambrogio 2010, §2]. Let

$$
\mathcal{L}=\operatorname{Loc}(\{\operatorname{cone}(f) \mid f \in R \backslash \mathfrak{p} \text { homogeneous }\}) .
$$

Then the Verdier quotient $Q: \mathcal{T} \rightarrow \mathcal{T} / \mathcal{L}=: \mathcal{T}_{\mathfrak{p}}$ has a fully faithful right adjoint, $I: \mathcal{T}_{\mathfrak{p}} \hookrightarrow \mathcal{T}$, and the functor $(-)_{\mathfrak{p}}$ can be defined to be the composite $(-)_{\mathfrak{p}}:=I \circ Q$. As $\mathcal{L}$ is generated by a tensor ideal of dualizable objects, we have $X_{\mathfrak{p}} \cong X \otimes \mathbf{1}_{\mathfrak{p}}$ for all $X \in \mathcal{T}$. Moreover, the unit $X \rightarrow X_{\mathfrak{p}}$ of the $(Q, I)$-adjunction induces a natural map $\operatorname{Hom}_{\mathcal{T}}^{*}(Y, X)_{\mathfrak{p}} \rightarrow \operatorname{Hom}_{\mathcal{T}}^{*}\left(Y, X_{\mathfrak{p}}\right)$ which is an isomorphism whenever $Y \in \mathcal{K}$ (see [Benson et al. 2011, Proposition 2.3] or [Dell' Ambrogio 2010, Theorem $2.33(\mathrm{~h})])$. In particular we have the identification

$$
\left(H^{*} X\right)_{\mathfrak{p}} \stackrel{\sim}{\longrightarrow} H^{*}\left(X_{\mathfrak{p}}\right)
$$


for all $X \in \mathcal{T}$. It follows also that the restriction of $Q$ to compact objects $X, Y \in \mathcal{K}$ agrees with $q_{\mathfrak{p}}$, so that we may identify $\mathcal{K}_{\mathfrak{p}}$ with the full subcategory $I\left(\mathcal{K}_{\mathfrak{p}}\right)$ of $\mathcal{T}$ (and thereby eliminate the slight ambiguity of the notation " $X_{\mathfrak{p}}$ ").

Recall the residue field objects $K(\mathfrak{p})$ defined in Section 3:

$$
K(\mathfrak{p}):=\mathbf{1}_{\mathfrak{p}} / /\left(f_{1}, \ldots, f_{n}\right) \simeq \mathbf{1}_{\mathfrak{p}} / / f_{1} \otimes \cdots \otimes \mathbf{1}_{\mathfrak{p}} / / f_{n} \in \mathcal{T}
$$

(as before, $f_{1}, \ldots, f_{n}$ denotes the chosen regular sequence of non-zero-divisors generating the prime $\mathfrak{p}$ ).

The main point of this section is that the crucial minimality result of Proposition 3.7 can be extended to localizing subcategories of $\mathcal{T}$, as we verify next.

Lemma 4.1. For every object $X \in \mathcal{T}$ and every $i \in\{1, \ldots, n\}$, each element $f$ of $\left(f_{1}, \ldots, f_{i}\right) \subset R$ acts as zero on $X_{\mathfrak{p}} / /\left(f_{1}, \ldots, f_{i}\right)$, i.e., $f \cdot X_{\mathfrak{p}} / /\left(f_{1}, \ldots, f_{i}\right)=0$. In particular, the $R$-module $H^{*}(X \otimes K(\mathfrak{p}))$ is a graded $k(\mathfrak{p})$-vector space.

Proof. Exactly the same as for Lemma 3.1 and Corollary 3.3. (Use that $X \otimes K(\mathfrak{p})=$ $X_{\mathfrak{p}} \otimes K(\mathfrak{p})$ to work inside the big $\mathfrak{p}$-local category $\mathcal{T}_{\mathfrak{p}}$.)

Proposition 4.2. For all $\mathfrak{p} \in \operatorname{Spec} R$ and $X \in \mathcal{T}$, the tensor product $X \otimes K(\mathfrak{p})$ decomposes into a coproduct of shifted copies of the residue field object:

$$
\coprod_{\alpha} \Sigma^{n_{\alpha}} K(\mathfrak{p}) \stackrel{\sim}{\longrightarrow} X \otimes K(\mathfrak{p}) .
$$

Proof. Exactly the same as for Proposition 3.6, using Lemma 4.1.

Proposition 4.3. For every $\mathfrak{p}$, the localizing subcategory $\operatorname{Loc}(K(\mathfrak{p}))$ of $\mathcal{T}$ is minimal, meaning that it contains no proper nonzero localizing subcategories.

Proof. This follows from Proposition 4.2 precisely as in the proof of Proposition 3.7, except that we cannot use Lemma 2.5 to show that $X \otimes K(\mathfrak{p}) \neq 0$ for every nonzero object $X \in \operatorname{Loc}(K(\mathfrak{p}))$. Instead, we may use the following argument.

First note that $X \otimes K(\mathfrak{q})=0$ for all $\mathfrak{q} \in$ Spec $R \backslash\{\mathfrak{p}\}$. Indeed, this property holds for $X=K(\mathfrak{p})$ by Lemma 4.1 (because if $\mathfrak{p} \neq \mathfrak{q}$ then some homogeneous element of $R$ must act on $K(\mathfrak{p}) \otimes K(\mathfrak{q})$ both as zero and invertibly) and is stable under taking coproducts and mapping cones (as the latter are preserved by $-\otimes K(\mathfrak{p})$ ); hence it must hold for all objects of $\operatorname{Loc}(K(\mathfrak{p}))$, as wished. Now combine this with Proposition 4.5 below.

Lemma 4.4. Let $M$ be any nonzero module, possibly infinitely generated, over a noetherian $\mathbb{Z}$-graded commutative ring $S$. Then there exists a minimal prime in $\operatorname{Supp}_{S} M:=\left\{\mathfrak{p} \in \operatorname{Spec} S \mid M_{\mathfrak{p}} \neq 0\right\}$, the big Zariski support of $M$.

Proof. If $M \neq 0$ then $M_{\mathfrak{p}} \neq 0$ for some prime $\mathfrak{p}$, so the support is not empty. Moreover, it suffices to prove the claim for the nonzero module $M_{\mathfrak{p}}$ over $S_{\mathfrak{p}}$, because a minimal prime of $\operatorname{Supp}_{S_{\mathfrak{p}}} M_{\mathfrak{p}}$ yields a minimal prime in $\operatorname{Supp}_{S} M$; hence we may 
assume that $S$ is local. By Zorn's lemma it suffices to show that in $\operatorname{Supp}_{S} M$ every chain of primes admits a minimum. Indeed, each such chain must stabilize, because a local commutative noetherian ring has finite Krull dimension. In the ungraded case, the latter is a well-known corollary of Krull's principal ideal theorem. A proof of the analogous result for graded rings can be found in [Bruns and Herzog 1993, Theorem 1.5.8] or [Park and Park 2011, Theorem 3.5].

Proposition 4.5. If an object $X \in \mathcal{T}$ is such that $X \otimes K(\mathfrak{p})=0$ for all $\mathfrak{p} \in \operatorname{Spec} R$ then $X=0$.

Proof. We prove the contrapositive. Assume that $X \neq 0$. Then $H^{*} X \neq 0$, hence for some $\mathfrak{p} \in \operatorname{Spec} R$ we must have $H^{*}\left(X_{\mathfrak{p}}\right)=\left(H^{*} X\right)_{\mathfrak{p}} \neq 0$ and therefore $X_{\mathfrak{p}} \neq 0$. By Lemma 4.4, we may choose a prime $\mathfrak{p}$ which is minimal among the primes with this property. Thus the big support of the $R$-module $H^{*} X_{\mathfrak{p}}$ consists precisely of the prime $\mathfrak{p}$. We are going to recursively show that $X_{i}:=X_{\mathfrak{p}} / /\left(f_{1}, \ldots, f_{i}\right)$ satisfies $\operatorname{Supp}_{R} H^{*} X_{i}=\{\mathfrak{p}\}$ for all $i \in\{1, \ldots, n\}$. Thus in particular $X \otimes K(\mathfrak{p})=X_{n} \neq 0$, which proves the proposition. We already know $\operatorname{Supp}_{R} H^{*} X_{0}=\{\mathfrak{p}\}$ for $X_{0}:=X_{\mathfrak{p}}$, and suppose we have shown that $\operatorname{Supp}_{R} H^{*} X_{i-1}=\{\mathfrak{p}\}$. The exact triangle

$$
\Sigma^{-\left|f_{i}\right|} X_{i-1} \stackrel{f_{i}}{\longrightarrow} X_{i-1} \longrightarrow X_{i} \longrightarrow \Sigma^{-\left|f_{i}\right|+1} X_{i-1}
$$

implies that $\operatorname{Supp}_{R} H^{*} X_{i} \subseteq\{\mathfrak{p}\}$. Hence $X_{i} \neq 0$ is equivalent to $\operatorname{Supp}_{R} H^{*} X_{i}=\{\mathfrak{p}\}$. By the triangle again, if $X_{i}=0$ were the case $f_{i}$ would act invertibly on $X_{i-1}$ and thus on $H^{*} X_{i-1}$. This implies $H^{*} X_{i-1}=\left(H^{*} X_{i-1}\right)\left[f_{i}^{-1}\right]$, and since $f_{i} \in \mathfrak{p}$ we would conclude that $\mathfrak{p} \notin \operatorname{Supp}_{R} H^{*} X_{i-1}$, in contradiction with the induction hypothesis. Therefore $X_{i} \neq 0$, as claimed.

Proof of Theorem 1.3. The result now follows easily from the machinery developed by Benson, Iyengar and Krause [Benson et al. 2008; 2011]. Indeed, by [Benson et al. 2011, Theorem 4.2], to obtain the claimed classification of localizing subcategories it suffices to verify that the action of $R$ stratifies $\mathcal{T}$. By definition, this means that the following two axioms are satisfied:

- The local-global principle: For every object $X \in \mathcal{T}$ we have the equality

$$
\operatorname{Loc}(X)=\operatorname{Loc}\left(\left\{\Gamma_{\mathfrak{p}} X \mid \mathfrak{p} \in \operatorname{Spec} R\right\}\right)
$$

of localizing subcategories of $\mathcal{T}$.

- Minimality: For every $\mathfrak{p} \in \operatorname{Spec} R$ the localizing subcategory $\Gamma_{\mathfrak{p}} \mathcal{T}$ of $\mathcal{T}$ is minimal or zero.

The functors $\Gamma_{\mathfrak{p}}: \mathcal{T} \rightarrow \mathcal{T}$ are introduced in [Benson et al. 2008], but we don't need to know how they are defined. In our context, i.e., where $\mathcal{T}$ is a tensor category and the action of $R$ is the canonical one of the central ring, the local-global principle always holds by [Benson et al. 2011, Theorem 7.2] (see also [Stevenson 2013, 
Theorem 6.8]). Moreover $\Gamma_{\mathfrak{p}} X=X \otimes \Gamma_{\mathfrak{p}} \mathbf{1}$ for all $X \in \mathcal{T}$, which implies that $\Gamma_{\mathfrak{p}} \mathcal{T}=\operatorname{Loc}\left(\Gamma_{\mathfrak{p}} \mathbf{1}\right)$ since $\mathcal{T}$ is generated by $\mathbf{1}$. Therefore the remaining minimality condition follows from Proposition 4.3, because $\operatorname{Loc}(K(\mathfrak{p}))=\operatorname{Loc}\left(\Gamma_{\mathfrak{p}} \mathbf{1}\right)$ by [Benson et al. 2011, Lemma 3.8 (2)] (indeed, by construction $K(\mathfrak{p})$ is a particular instance of the objects collectively denoted by $\mathbf{1}(\mathfrak{p})$ in [loc. cit.]). This establishes the first bijection in Theorem 1.3.

The claimed identification of the Benson-Iyengar-Krause support, $\operatorname{supp}_{R} X=$ $\left\{\mathfrak{p} \in \operatorname{Spec} R \mid X \otimes \Gamma_{\mathfrak{p}} \mathbf{1} \neq 0\right\}$, with the set $\{\mathfrak{p} \in \operatorname{Spec} R \mid X \otimes K(\mathfrak{p}) \neq 0\}$ is an easy consequence of the equality $\operatorname{Loc}(K(\mathfrak{p}))=\operatorname{Loc}\left(\Gamma_{\mathfrak{p}} 1\right)$ mentioned above.

It remains to verify the moreover part of Theorem 1.3. Let us begin by noting that, if $X \in \mathcal{K}$ is a compact object, we have

$$
\operatorname{supp}_{R} X=\operatorname{supp}_{R} H^{*} X=\operatorname{Supp}_{R} H^{*} X
$$

by [Benson et al. 2008, Theorem 5.5(1)] and Lemma 2.1. Now let $\mathcal{L} \subseteq \mathcal{T}$ be such that $\mathcal{L}=\operatorname{Loc}(\mathcal{L} \cap \mathcal{K})$. Then

$$
\bigcup_{X \in \mathcal{L}} \operatorname{supp}_{R} X=\bigcup_{X \in \mathcal{L} \cap \mathcal{K}} \operatorname{supp}_{R} X=\bigcup_{X \in \mathcal{L} \cap \mathcal{K}} \operatorname{Supp}_{R} H^{*} X
$$

by (4.6), and the latter is a specialization closed subset of the spectrum. Conversely, if $S \subseteq \operatorname{Spec} R$ is specialization closed the corresponding localizing subcategory $\left\{X \in \mathcal{T} \mid \operatorname{supp}_{R} X \subseteq S\right\}$ is generated by compact objects by [Benson et al. 2008, Theorem 6.4], hence $\mathcal{L}=\operatorname{Loc}(\mathcal{L} \cap \mathcal{K})$. This concludes the proof of the theorem.

It is well known that the assignments $\mathcal{C} \mapsto \operatorname{Loc}(\mathcal{C})$ and $\mathcal{L} \mapsto \mathcal{L} \cap \mathcal{K}$ are mutually inverse bijections between thick subcategories $\mathcal{C} \subseteq \mathcal{K}$ and localizing subcategories $\mathcal{L} \subseteq \mathcal{T}$ which are generated by compact objects of $\mathcal{T}$ (see [Neeman 1992b]). Together with (4.6), this shows how to deduce the classification of thick subcategories of Corollary 1.2 from Theorem 1.3.

Finally, there are several ways to derive the telescope conjecture of Corollary 1.4 from the previous results. For instance, we may proceed as in [Benson et al. 2011, $\S 6.2]$.

Remark 4.7. Using the theory of coherent functors, Benson, Iyengar and Krause have recently developed in [Benson et al. 2015] an analogue of their stratification theory of compactly generated categories that can be applied to general essentially small triangulated categories. Their theory, and more specifically [Benson et al. 2015, Theorem 7.4], provides an alternative way to derive Theorem 1.1 from Proposition 3.7.

The case of commutative dg algebras. We still owe readers a proof of Theorem 1.6. Let $A$ be a commutative dg algebra and let $D(A)$ be the derived category of (left, 
say) $\operatorname{dg}-A$-modules. The following elementary fact was pointed out to us by the referee.

Lemma 4.8. Every $f \in H^{*} A$ acts as zero on its own mapping cone $C(f)$.

Proof. A (homogeneous) element $f \in H^{*} A$ of degree $|f|=-n$ is (represented by) a morphism $f: \Sigma^{n} A \rightarrow A$ of left $\operatorname{dg}-A$-modules. Let us write s $a(a \in A)$ for a generic element of degree $|a|-1$ in the suspension $\mathrm{s} A:=\Sigma A$; here we use the Koszul sign convention and treat s as a symbol of degree -1 . The cone $C(f)$ has elements $\left(a, \mathrm{~s}^{n+1} b\right)$ (for $a, b \in A$ ). Then $f$ acts on $C(f)$ by a morphism $\mathrm{s}^{n} C(f) \rightarrow C(f)$ which, under the isomorphism $\mathrm{s}^{n} C(f) \cong C\left(\mathrm{~s}^{n} f\right)$, is written as follows:

$$
g: C\left(\mathrm{~s}^{n} f\right) \rightarrow C(f), \quad g\left(\mathrm{~s}^{n} a, \mathrm{~s}^{2 n+1} b\right)=\left(f\left(\mathrm{~s}^{n} a\right), \mathrm{s}^{n+1} f\left(\mathrm{~s}^{n} b\right)\right)
$$

(recall that the suspension $\mathrm{s} h: \mathrm{s} B \rightarrow \mathrm{s} C$ of a morphism $h: B \rightarrow C$ is given by $(\mathrm{s} h)(\mathrm{s} b)=\mathrm{s}(h(b)))$. With these notations, the map $H: C\left(\mathrm{~s}^{n} f\right) \rightarrow C(f)$ defined by $H\left(\mathrm{~s}^{n} a, \mathrm{~s}^{2 n+1} b\right):=\left(0, \mathrm{~s}^{n+1} a\right)$ is easily seen to satisfy $H(t x)=(-1)^{|t|} t H(x)$ (for $\left.t \in A, x \in C\left(\mathrm{~s}^{n} f\right)\right)$ and $d H+H d=-g$; in other words, $H$ is a homotopy $g \sim 0$ defined over $A$.

As noted in Remark 3.5, Lemma 3.1 was the only place in all of our arguments where we made use of the hypothesis that $R$ is concentrated in even degrees and that in the regular sequences we may choose the elements to be non-zero-divisors. But if we consider the example $\mathcal{K}:=D(A)^{c}, \mathcal{T}:=D(A)$ and $R:=H^{*} A$, we see immediately that the conclusion of the lemma also follows from the above result (Lemma 4.8). Hence in this case we can get rid of the extra hypotheses, while the rest of our arguments go through unchanged. This proves Theorem 1.6.

Indeed, in general in condition (b) of Theorem 1.1 we could similarly renounce the evenness of $R$ if we substitute the requirement that all elements $f_{i}$ of the regular sequences be non-zero-divisors with the requirement that $f_{i} \cdot \mathbf{1} / / f_{i}=0$.

\section{Acknowledgements}

We are very grateful to Paul Balmer and an anonymous referee for their useful comments, and to the referee for suggesting Theorem 1.6. We also thank Joseph Chuang for the reference [Mathew 2016].

\section{References}

[Balmer 2005] P. Balmer, "The spectrum of prime ideals in tensor triangulated categories", J. Reine Angew. Math. 588 (2005), 149-168. MR 2196732 Zbl 1080.18007

[Balmer 2007] P. Balmer, "Supports and filtrations in algebraic geometry and modular representation theory”, Amer. J. Math. 129:5 (2007), 1227-1250. MR 2354319 Zbl 1130.18005 
[Balmer 2010a] P. Balmer, "Spectra, spectra, spectra - tensor triangular spectra versus Zariski spectra of endomorphism rings", Algebr. Geom. Topol. 10:3 (2010), 1521-1563. MR 2661535 Zbl 1204.18005

[Balmer 2010b] P. Balmer, “Tensor triangular geometry”, pp. 85-112 in Proceedings of the International Congress of Mathematicians, vol. II, edited by R. Bhatia et al., Hindustan Book Agency, New Delhi, 2010. MR 2827786 Zbl 1204.18004

[Benson et al. 2008] D. Benson, S. B. Iyengar, and H. Krause, "Local cohomology and support for triangulated categories”, Ann. Sci. Éc. Norm. Supér. (4) 41:4 (2008), 573-619. MR 2489634 Zbl 1171.18007

[Benson et al. 2011] D. Benson, S. B. Iyengar, and H. Krause, "Stratifying triangulated categories", J. Topol. 4:3 (2011), 641-666. MR 2832572 Zbl 1239.18013

[Benson et al. 2015] D. Benson, S. B. Iyengar, and H. Krause, "A local-global principle for small triangulated categories”, Math. Proc. Cambridge Philos. Soc. 158:3 (2015), 451-476. MR 3335421

[Bruns and Herzog 1993] W. Bruns and J. Herzog, Cohen-Macaulay rings, Cambridge Studies in Advanced Mathematics 39, Cambridge University Press, Cambridge, 1993. MR 1251956 Zbl 0788.13005

[Dell'Ambrogio 2010] I. Dell' Ambrogio, “Tensor triangular geometry and $K K$-theory”, J. Homotopy Relat. Struct. 5:1 (2010), 319-358. MR 2812924 Zbl 1278.19010

[Dell' Ambrogio and Tabuada 2012] I. Dell'Ambrogio and G. Tabuada, "Tensor triangular geometry of non-commutative motives", Adv. Math. 229:2 (2012), 1329-1357. MR 2855096 Zbl 1250.18012

[Goto and Yamagishi 1983] S. Goto and K. Yamagishi, "Finite generation of Noetherian graded rings”, Proc. Amer. Math. Soc. 89:1 (1983), 41-44. MR 706507 Zbl 0528.13015

[Hochster 1969] M. Hochster, "Prime ideal structure in commutative rings", Trans. Amer. Math. Soc. 142 (1969), 43-60. MR 0251026 Zbl 0184.29401

[Keller 1994] B. Keller, "A remark on the generalized smashing conjecture”, Manuscripta Math. 84:2 (1994), 193-198. MR 1285956 Zbl 0826.18004

[Mandell 2012] M. A. Mandell, "The smash product for derived categories in stable homotopy theory", Adv. Math. 230:4-6 (2012), 1531-1556. MR 2927347 Zbl 1246.55010

[Mathew 2015] A. Mathew, "A thick subcategory theorem for modules over certain ring spectra", Geom. Topol. 19:4 (2015), 2359-2392. MR 3375530 Zbl 06472920

[Mathew 2016] A. Mathew, "Residue fields for a class of rational $\mathbf{E}_{\infty}$-rings and spectra", J. Pure Appl. Algebra (online publication July 2016).

[Neeman 1992a] A. Neeman, "The chromatic tower for $D(R)$ ", Topology 31:3 (1992), 519-532. MR 1174255 Zbl 0793.18008

[Neeman 1992b] A. Neeman, "The connection between the $K$-theory localization theorem of Thomason, Trobaugh and Yao and the smashing subcategories of Bousfield and Ravenel", Ann. Sci. École Norm. Sup. (4) 25:5 (1992), 547-566. MR 1191736 Zbl 0868.19001

[Park and Park 2011] C. H. Park and M. H. Park, "Integral closure of a graded Noetherian domain", J. Korean Math. Soc. 48:3 (2011), 449-464. MR 2815884 Zbl 1218.13001

[Shamir 2012] S. Shamir, "Stratifying derived categories of cochains on certain spaces", Math. Z. 272:3-4 (2012), 839-868. MR 2995142 Zbl 1271.55010

[Stevenson 2013] G. Stevenson, "Support theory via actions of tensor triangulated categories", $J$. Reine Angew. Math. 681 (2013), 219-254. MR 3181496 Zbl 1280.18010

[Thomason 1997] R. W. Thomason, "The classification of triangulated subcategories", Compositio Math. 105:1 (1997), 1-27. MR 1436741 Zbl 0873.18003 
Received November 16, 2015. Revised May 9, 2016.

IVO DELL'AMBRogio

Laboratoire de Mathématiques Paul Painlevé UNIVERSITÉ DE LILLE 1

BÂT. M2

Cité SCIENTIFIQUe

59665 VILlENEUVE D’ AsCQ CÉDEX

FRANCE

ivo.dellambrogio@math.univ-lille1.fr

DONALD STANLEY

DEPARTMENT OF MATHEMATICS AND STATISTICS

UNIVERSITY OF REGINA

COLlege West 307.14

3737 WASCANA PARKWAY

REGINA, SK S4S 0A2

CANADA

donald.stanley@uregina.ca 


\title{
PACIFIC JOURNAL OF MATHEMATICS
}

Founded in 1951 by E. F. Beckenbach (1906-1982) and F. Wolf (1904-1989)

$$
\text { msp.org/pjm }
$$

\section{EDITORS}

\author{
Don Blasius (Managing Editor) \\ Department of Mathematics \\ University of California \\ Los Angeles, CA 90095-1555 \\ blasius@math.ucla.edu
}

\author{
Paul Balmer \\ Department of Mathematics \\ University of California \\ Los Angeles, CA 90095-1555 \\ balmer@math.ucla.edu \\ Robert Finn \\ Department of Mathematics \\ Stanford University \\ Stanford, CA 94305-2125 \\ finn@math.stanford.edu \\ Sorin Popa \\ Department of Mathematics \\ University of California \\ Los Angeles, CA 90095-1555 \\ popa@math.ucla.edu
}

\author{
Vyjayanthi Chari \\ Department of Mathematics \\ University of California \\ Riverside, CA 92521-0135 \\ chari@math.ucr.edu \\ Kefeng Liu \\ Department of Mathematics \\ University of California \\ Los Angeles, CA 90095-1555 \\ liu@math.ucla.edu \\ Igor Pak \\ Department of Mathematics \\ University of California \\ Los Angeles, CA 90095-1555 \\ pak.pjm@gmail.com \\ Paul Yang \\ Department of Mathematics \\ Princeton University \\ Princeton NJ 08544-1000 \\ yang@math.princeton.edu
}

\section{PRODUCTION}

Silvio Levy, Scientific Editor, production@msp.org

\section{SUPPORTING INSTITUTIONS}

ACADEMIA SINICA, TAIPEI

CALIFORNIA INST. OF TECHNOLOGY

STANFORD UNIVERSITY

UNIV. OF BRITISH COLUMBIA

UNIV. OF CALIFORNIA, BERKELEY

UNIV. OF CALIFORNIA, DAVIS

UNIV. OF CALIFORNIA, LOS ANGELES

UNIV. OF CALIFORNIA, RIVERSIDE

UNIV. OF CALIFORNIA, SAN DIEGO

UNIV. OF CALIF., SANTA BARBARA
KEIO UNIVERSITY

MATH. SCIENCES RESEARCH INSTITUTE

NEW MEXICO STATE UNIV.

OREGON STATE UNIV.
Daryl Cooper

Department of Mathematics

University of California

Santa Barbara, CA 93106-3080 cooper@math.ucsb.edu

Jiang-Hua Lu

Department of Mathematics

The University of Hong Kong

Pokfulam Rd., Hong Kong

jhlu@maths.hku.hk

$$
\text { Jie Qing }
$$

Department of Mathematics

University of California

Santa Cruz, CA 95064

qing@ cats.ucsc.edu

\author{
UNIV. OF CALIF., SANTA CRUZ \\ UNIV. OF MONTANA \\ UNIV. OF OREGON \\ UNIV. OF SOUTHERN CALIFORNIA \\ UNIV. OF UTAH \\ UNIV. OF WASHINGTON \\ WASHINGTON STATE UNIVERSITY
}

These supporting institutions contribute to the cost of publication of this Journal, but they are not owners or publishers and have no responsibility for its contents or policies.

See inside back cover or msp.org/pjm for submission instructions.

The subscription price for 2016 is US $\$ 440 /$ year for the electronic version, and \$600/year for print and electronic.

Subscriptions, requests for back issues and changes of subscriber address should be sent to Pacific Journal of Mathematics, P.O. Box 4163, Berkeley, CA 94704-0163, U.S.A. The Pacific Journal of Mathematics is indexed by Mathematical Reviews, Zentralblatt MATH, PASCAL CNRS Index, Referativnyi Zhurnal, Current Mathematical Publications and Web of Knowledge (Science Citation Index).

The Pacific Journal of Mathematics (ISSN 0030-8730) at the University of California, c/o Department of Mathematics, 798 Evans Hall \#3840, Berkeley, CA 94720-3840, is published twelve times a year. Periodical rate postage paid at Berkeley, CA 94704, and additional mailing offices. POSTMASTER: send address changes to Pacific Journal of Mathematics, P.O. Box 4163, Berkeley, CA 94704-0163.

PJM peer review and production are managed by EditFLOW ${ }^{\circledR}$ from Mathematical Sciences Publishers.

PUBLISHED BY

\section{I. mathematical sciences publishers}

nonprofit scientific publishing

http://msp.org/

(C) 2016 Mathematical Sciences Publishers 


\section{PACIFIC JOURNAL OF MATHEMATICS}

Volume $285 \quad$ No. $1 \quad$ November 2016

Iwahori-Hecke algebras for Kac-Moody groups over local fields

Nicole BARDY-PANSE, StÉPHANE GAUSSENT and GuY

ROUSSEAU

A classification of spherical conjugacy classes

MAURo Costantini

Affine weakly regular tensor triangulated categories

IVo DELL'AMBROGIO and DONALD STANLEY

Involutive automorphisms of $N_{\circ}^{\circ}$-groups of finite Morley rank

ADRIEN DELORO and ÉRIC JALIGOT

Schur-Weyl duality for Deligne categories, II: The limit case

INNA ENTOVA AIZENBUD

A generalization of the Greene-Krantz theorem for the semicontinuity 225 property of automorphism groups

JAE-CHEON JOO

Gradient estimates for a nonlinear Lichnerowicz equation under general geometric flow on complete noncompact manifolds

LIANG ZHAO and SHOUWEN FANG 